FORMATION Formation emploi

Revue française de sciences sociales

100 | octobre-décembre 2007

De la formation professionnelle en Suisse

\title{
Les enjeux identitaires de la formation professionnelle duale en Suisse : un tableau en demi-teinte
}

The question of identity in the dual Swiss vocational training system: a contrasting picture

Identitätsfragen in der dualen Berufsausbildung in der Schweiz: ein Bild mit einigen Schatten

Jonas Masdonati, Nadia Lamamra, Benoît Gay-des-Combes et Jacqueline De Puy

\section{OpenEdition}

Journals

Édition électronique

URL : http://journals.openedition.org/formationemploi/1253

DOI : 10.4000/formationemploi.1253

ISSN : 2107-0946

Éditeur

La Documentation française

Édition imprimée

Date de publication : 1 octobre 2007

Pagination : 15-29

ISSN : 0759-6340

Référence électronique

Jonas Masdonati, Nadia Lamamra, Benoît Gay-des-Combes et Jacqueline De Puy, « Les enjeux identitaires de la formation professionnelle duale en Suisse : un tableau en demi-teinte », Formation emploi [En ligne], 100 | octobre-décembre 2007, mis en ligne le 01 octobre 2009, consulté le 30 octobre 2020. URL : http://journals.openedition.org/formationemploi/1253 ; DOI : https://doi.org/ 10.4000/formationemploi.1253 


\section{DOSSIER}

\section{Les enjeux identitaires de la formation professionnelle duale en Suisse : un tableau en demi-teintel}

Par Jonas Masdonati, Nadia Lamamra, Benoît Gay-des-Combes, Jacqueline De Puy*

L'apprentissage dual peut permettre aux jeunes d'affronter la transition entre le monde scolaire et celui du travail, favorisant ainsi la transition entre une identité d'élève et celle de professionnel. Cependant, les témoignages recueillis révèlent que l'apprentissage dual présente plusieurs limites non négligeables.

La question des avantages et inconvénients de l'apprentissage dual est d'une grande actualité dans les débats autour de la formation professionnelle en Suisse et en Europe, notamment en termes de comparaison internationale (Kirpal, 2006). L'originalité de notre démarche consiste d'une part à aborder cette question à la fois dans une perspective sociologique et psychosociale et d'autre part à la centrer sur les apprenants et leur développement identitaire.

\footnotetext{
${ }^{1}$ L'article s'appuie sur les résultats intermédiaires de deux recherches menées à l'Institut fédéral des hautes études en formation professionnelle (IFFP), résultats qui n'ont pas encore fait l'objet de publications, mais qui ont été présentés séparément dans des congrès. Les projets sont financés par l'Office fédéral de la formation professionnelle et de la technologie (OFFT).
}

* Jonas Masdonati est collaborateur scientifique, licencié en psychologie et docteur en sciences de l'éducation. Ses domaines d'investigation concernent la transition écoletravail, l'orientation scolaire et professionnelle, la construction identitaire, les jeunes en difficulté, la résilience. II a notamment publié : MasdonatiJ. (2007), La transition entre école et monde du travail : Préparer les jeunes à l'entrée en formation professionnelle, Bern: Peter Lang. Oser F., Gamboni E., Masdonati J. \& Düggeli A. (2005), «Die Zeitbombe des "dummen" Schülers: Eine Interventionsstudie zur Erhöhung von Resilienz bei drohender oder erfahrener Erwerbslosigkeit unterqualifizierter Jugendlicher », in Chaponnière M., Flückiger Y., Hotz-Hart B., Osterwalder F., Sheldon G. \& Weber K. (Eds.), Forum formation et emploi : La relation formation-emploi en question, Vol. 1, pp. 173181, Zürich; Chur: Verlag Rüegger. 
Nadia Lamamra est collaboratrice scientifique, licenciée en lettres (histoire contemporaine). Ses domaines d'investigation sont le genre dans la formation professionnelle, les rapports sociaux de sexe, la migration, les jeunes en difficulté. Elle a notamment publié : Ducret V. \& Lamamra N. (2005), Pour intégrer le genre dans la formation professionnelle. Guide à l'usage des formateurs et formatrices, Lausanne/Genève: ISPFP/Le deuxième Observatoire. Lamamra N. \& Masdonati J. (sous presse), Jeunes en difficulté dans la formation professionnelle: Une question identitaire? Actes du 14e Congrès de l'Association internationale de psychologie du travail de langue française, Hammamet.

Benoît Gay-des-Combes est collaborateur scientifique, licencié en sciences sociales. Ses domaines de recherche ont trait aux aspects sociologiques de la formation professionnelle, aux politiques sociales, aux réformes de l'État social. Il a notamment publié : Bertozzi F., Bonoli G. \& Gay-des-Combes B. (2004), La réforme de l'État social en Suisse / vieillissement, emploi, conflit travaitfamille, coll. "Le savoir suisse » $n^{\circ} 27$, Lausanne, Presses polytechniques et universitaires romandes (PPUR). De PuyJ., Castelli-Dransart D. A., Gay-des-Combes B., Perriard V. \& Zbinden-Sapin V. (2006), "Young Trainees and Professional Identity in Switzerland: Pride or Prejudice » in Sociological Abstracts, 2006500507.

Jacqueline De Puy est maître de recherche, licenciée en sociologie et docteure en travail social et politiques sociales. Ses domaines d'investigation touchent aux aspects sociologiques de la formation professionnelle, à la prévention et promotion de la santé, aux compétences sociales et personnelles. Elle a notamment publié : De PuyJ. (2004), " Un outil pour prévenir les violences et promouvoir les compétences relationnelles dans les fréquentations amoureuses des jeunes: Sortir ensemble et se respecter », Journal européen d'éducation sociale, 6. De PuyJ., CastelliDransart D. A., Gay-des-Combes B., Perriard V. \& ZbindenSapin V. (2006), "Young Trainees and Professional Identity in Switzerland: Pride or Prejudice " in Sociological Abstracts, 2006 S00507.

Cet article soulève un questionnement, situé à l'intersection de deux recherches en cours à l'Institut fédéral des hautes études en formation professionnelle (IFFP), sur les liens entre formation professionnelle duale et identité des apprenants ( $c f$. encadré 1), et ce à partir de l'analyse de récits de jeunes en début de formation et d'autres en situation de rupture. Tout d'abord, le contexte et la spécificité de la formation professionnelle duale en Suisse sont brièvement posés. Puis, à partir de travaux existants et d'une analyse préliminaire de nos données, une discussion est menée sur les avantages et inconvénients de ce système de formation, en regard de la construction identitaire des apprenants. ${ }^{2}$

\section{Encadré 1 Éléments de terminologie}

En Suisse, la formation professionnelle duale correspond à une formation par alternance. Celle-ci combine cours théoriques dans une école professionnelle et apprentissage pratique du métier en entreprise. On parle de "formation triale " lorsque s'y ajoutent des cours interentreprises. Quelques éclaircissements terminologiques s'imposent :

- Les termes «formation professionnelle duale » et " apprentissage dual » seront utilisés indistinctement dans le cadre de cet article. Ils sont considérés comme synonymes de « formation par alternance » ou encore de "formation professionnelle initiale duale». - Par «apprenants", nous entendons les jeunes en formation professionnelle duale (appelés apprentis avant la loi de 2004). Les «formateurs » (appelés maîtres d'apprentissage avant la loi de 2004) sont les personnes qui, dans le cadre de l'apprentissage dual, s'occupent de la formation pratique des apprenants en entreprise.

- Les " enseignants " s'occupent de la formation générale et théorique en école professionnelle.

- Nous appelons «experts » les personnes reconnues comme spécialistes et expérimentées dans le champ de la formation professionnelle ladministrateurs, directeurs d'école professionnelle, etc.)

\footnotetext{
${ }^{2} \mathrm{Au}$ moment où paraît ce dossier sur la formation professionnelle en Suisse, les résultats définitifs de nos deux recherches ne sont pas encore disponibles. Cependant, l'intention de notre démarche est de mener une première réflexion transversale aux deux projets.
} 


\section{UN SYSTÈME DE FORMATION LIÉ AU MARCHÉ DE L'EMPLOI}

En Suisse, la formation professionnelle reste aujourd'hui encore la voie de formation post-obligatoire privilégiée. Pour l'année 2005, environ troisquarts des personnes ayant débuté une formation du secondaire II (enseignement post-obligatoire) l'ont fait dans la formation professionnelle. À l'intérieur de cette filière, près de $80 \%$ des jeunes optent pour la formation professionnelle duale (OFFT - Office fédéral de la formation professionnelle et de la technologie, 2006). Dans ce système dit dual, qui s'inscrit dans la même tradition que le modèle allemand (Troger, 2003), la formation professionnelle est étroitement liée au marché de l'emploi, puisque d'une part, les lieux de formation sont en grande partie des entreprises soumises aux impératifs de rentabilité et de productivité et, d'autre part, les personnes issues d'une telle formation doivent pouvoir être immédiatement «employables » sur le marché du travail. Ce lien explique que les mutations intervenues dans le marché de l'emploi influencent très directement le système de formation professionnelle. Ceci d'autant plus que la politique de formation professionnelle est négociée entre trois partenaires : la Confédération, les cantons et les organisations du monde du travail (Dubs, 2006).

Ces dernières décennies, le monde du travail des sociétés post-industrielles a connu de profondes transformations (Castel, 1995 ; Rifkin, 1996), notamment par la tertiarisation de l'emploi, l'innovation technologique et la disparition de certains métiers traditionnels, remplacés par des nouvelles professions issues du regroupement de professions existantes et dont les formations sont souvent plus exigeantes et plus longues ${ }^{3}$. Ces mutations transforment le marché du travail, qui requiert plus que jamais des personnes polyvalentes et flexibles, pouvant s'adapter aux évolutions parfois rapides des

\footnotetext{
${ }^{3}$ Le processus est en cours et les associations professionnelles travaillent actuellement sur les cadres législatifs (ordonnances de formation) accompagnant ces nouvelles professions. $\grave{A}$ titre d'exemple, la formation plus exigeante de poly-mécanicien regroupe et remplace huit anciennes professions, tandis que la durée des formations de vendeur et d'employé de bureau est passée de deux à trois ans.
}

professions, tout en étant spécialisées et fiables dans leur champ de compétences (Poglia, 1999 ; Sennett, 2000).

Pour répondre à ces exigences, la formation professionnelle suisse a, elle-aussi, entamé une profonde transformation cette dernière décennie. Une augmentation générale du niveau de formation, signe d'une « intellectualisation» de la formation professionnelle, a répondu aux mutations évoquées ci-dessus (Moser, 2004 ; Poglia, 1999). L'entrée en vigueur, le

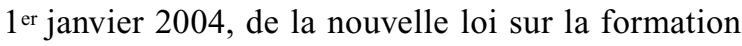
professionnelle, confirme cette uniformisation par le haut. Par exemple, celle-ci met en place des

Encadré 2
L'enseignement post-obligatoire
en Suisse
L'enseignement post-obligatoire en Suisse,
aussi appelé « Secondaire II ", intervient dès
15 ans et offre trois possibilités de formation
ou d'étude : la filière professionnelle, la
filière de culture générale et la filière
gymnasiale :
- La filière professionnelle est orientée vers la
pratique, se déroule en entreprise ou en
école de métiers et peut s'échelonner sur
deux ans (attestation fédérale de formation
professionnelle) ou sur trois ou quatre ans
(certificat fédéral de capacité). Les personnes
sortant de cette filière peuvent ensuite entrer
dans le monde du travail ou poursuivre leur
formation dans une École supérieure ou une
Haute école spécialisée.
- La filière de culture générale, qui se
déroule en école, combine un enseignement
de culture générale avec des cours spéci-
fiques permettant de poursuivre une forma-
tion professionnelle dans les domaines de la
santé ou du social, notamment.
- La filière gymnasiale (lycée) permet
d'acquérir, en école, les connaissances
requises pour l'entrée à l'université, dans une
École polytechnique fédérale ou dans une
Haute école pédagogique (http://www.orien-
tation.ch).


passerelles («maturité professionnelle ») permettant d'accéder à des formations supérieures, et, dans le même temps, elle élève le niveau d'exigences des formations destinées aux jeunes en difficulté ( formation professionnelle initiale en deux ans $»)^{4}$. $\mathrm{Si}$ ces réformes préparent les futurs travailleurs à relever les nouveaux défis du monde professionnel, elles risquent d'exclure un nombre croissant de jeunes de cet univers (Meyer, 2003 ; Moser, 2004).

Simultanément, le décalage entre le nombre de jeunes qui cherchent un apprentissage et le nombre de places vacantes persiste (Hanhart, 2006)5. En outre, l'offre de places d'apprentissage ne correspond pas forcément aux désirs des jeunes (LINK, 2005 ; Rastoldo, 2006). Dès lors, de nombreux jeunes sont soit obligés d'apprendre un métier qu'ils n'avaient pas choisi, soit ne trouvent pas de place dans une entreprise formatrice et sont momentanément exclus du marché du travail, dans des solutions dites «transitoires » (Meyer, 2004).

Enfin, même les jeunes qui ont déjà entamé une formation professionnelle semblent influencés par ce contexte précaire. En effet, le risque de chômage et la rupture de contrats d'apprentissage sont autant d'éléments qui pèsent sur eux. Le taux élevé d'interruptions d'apprentissage dual (Stalder \& Schmid, 2006a) ${ }^{6}$ peut être vu comme un signe tangible de cette pression.

\footnotetext{
${ }^{4} \mathrm{La}$ formation professionnelle initiale en deux ans remplace l'ancienne formation élémentaire, qui était basée sur la mise en place d'objectifs de formation individualisés. Si les deux modèles sont destinés aux jeunes en difficultés, la nouvelle formation implique des exigences plus élevées que la précédente, notamment parce qu'elle introduit des objectifs de formation uniformisés - et donc non adaptés aux besoins et possibilités de chaque apprenant. La formation professionnelle initiale en deux ans a cependant l'avantage de fournir aux apprenants un encadrement individuel spécialisé et des appuis pédagogiques censés les aider à atteindre ces objectifs.

${ }^{5}$ L'Union syndicale suisse estime par exemple qu'il manque aujourd'hui 10000 places d'apprentissage (Sigerist \& Schwaab, 2006).

${ }^{6}$ Même si aucune statistique n'existe au niveau national, des données cantonales sont parfois disponibles. De manière très générale, on observe des taux de rupture d'apprentissage allant de 10 à $30 \%$ selon les régions et la méthodologie utilisée. Malgré les différences cantonales, ces taux sont stables depuis une dizaine d'années. À titre d'exemples, les taux de résiliation de contrats d'apprentissage vont de $12 \%$ (Neuchâtel 2004/2005), à $13 \%$ (Vaud 2001/ 2002 ) et à $24 \%$ (Berne 2004/2005), voire $29 \%$ (Genève 2000/ 2001) (Stalder \& Schmid, 2006a, 2006b).
}

\section{DES ENJEUX IDENTITAIRES PEU EXPLORÉS}

Dans un contexte où la flexibilité est requise et où la non-linéarité caractérise le déroulement des trajectoires professionnelles, il est pertinent de questionner les notions d'identité et de socialisation professionnelles. En effet, qu'adviendra-t-il si chacun doit «changer régulièrement d'activité, d'emploi, de compétence, de réseau? Que deviendra alors son identité professionnelle, partie plus ou moins centrale de son identité personnelle? [...] Sera-t-elle, pour eux, une série indéfinie de crises à surmonter, à gérer? »(Dubar, 2000, pp. 127-128). Au vu de la spécificité du système de formation professionnelle suisse, ce questionnement doit également intervenir au niveau de l'apprentissage dual. Cela d'autant plus si l'on considère qu'il constitue une première instance de socialisation professionnelle, c'est-à-dire de transmission, explicite ou implicite, de rôles, de statuts et de valeurs associés à des professions ou des champs professionnels (Ruano-Borbalan 2004; Zittoun, 2006).

L'importance de la problématique identitaire en formation professionnelle duale est accentuée par le fait que les apprenants peuvent démarrer leur apprentissage dual dès l'âge de 15 ans (la moyenne se situant à 17 ans). Ainsi, durant la période de l'apprentissage, la construction d'une identité professionnelle s'opère parallèlement à la construction d'une identité personnelle. Cette tranche d'âge correspond en effet à l'adolescence, c'est-à-dire à un moment du développement durant lequel la personne cherche à se définir en tant que personne et à se positionner au sein de la société (Erikson, 1972; Flammer \& Alsaker, 2002 ; Schulenberg, Maggs \& Hurrelmann, 1997). Ainsi, ces années d'apprentissage influencent-elles sans doute considérablement la trajectoire professionnelle et personnelle des jeunes (Cohen-Scali, 2001a).

Toutefois, le rôle de la formation professionnelle duale dans les processus de développement identitaire des apprenants est encore peu approfondi. Ce système de formation présente des atouts, mais également des failles en termes d'apports identitaires pour les jeunes (Cohen-Scali, 2000). L'auteur estime 
en effet que la formation duale facilite l'insertion professionnelle des jeunes, leur permet de se mobiliser pour un projet professionnel et favorise leur acculturation aux valeurs et aux normes de l'entreprise. Cependant, la mobilisation des jeunes n'est possible que lorsque ceux-ci ont un projet professionnel établi, sont impliqués dans les activités mises en place par l'entreprise et parviennent à créer des liens avec les personnes travaillant au sein de l'organisation. En outre, la construction d'une identité professionnelle peut être difficile lorsque les tâches confiées aux apprenants sont disqualifiantes ou peu formatrices et lorsque leur rôle n'est pas clair. Enfin, l'identité professionnelle est tributaire de la recon- naissance économique, interpersonnelle et sociale de l'apprenant par l'entreprise et ses membres. Ainsi, si l'apprenant n'est pas reconnu comme acteur productif et comme individu à part entière et si son statut n'est pas clairement défini, la construction de son identité professionnelle risque d'être lacunaire.

Dès lors, sur quelles fondations peut se construire l'identité professionnelle des jeunes en apprentissage dual et quel rôle jouent les écoles professionnelles et les entreprises formatrices dans la transmission d'une identité professionnelle? (De Puy, Castelli-Dransart, Gay-des-Combes, Perriard, \& Zbinden Sapin, 2006 ; Rochat \& Lamamra, 2004).

\section{Encadré 3 \\ Deux recherches sur l'identité des apprenants}

Deux recherches actuellement en cours s'attachent à investiguer les liens entre formation professionnelle et identité des apprenants. Ces études ont également pour but de déboucher sur des propositions concrètes destinées aux enseignants, formateurs et autres acteurs de la formation professionnelle.

Étude E1 : L'identité professionnelle au sein de la formation professionnelle initiale. Représentations collectives de deux professions parmi les apprenants, enseignants et formateurs.

Equipe du projet : Dolores Angela Castelli-Dransart, Valérie Perriard et Véronique Zbinden Sapin, Haute école fribourgeoise de travail social (HEF-TS); Jacqueline De Puy et Benoît Gay-des-Combes, Institut fédéral des hautes études en formation professionnelle (IFFP).

Menée conjointement avec la HEF-TS, la première recherche se propose de travailler sur la notion d'identité professionnelle valorisée et valorisante, pouvant être revendiquée et négociée de manière positive par de futurs professionnels. Elle cherche ainsi à comprendre comment se définit "un professionnel » dans deux corps de métier (poly-mécanicien et assistant socio-éducatif), et ce à partir des représentations de différents acteurs, tels que des jeunes en formation, des formateurs en entreprise, ou encore des enseignants des écoles professionnelles. Au total, 134 personnes ont été consultées par le biais d'entretiens semi-directifs d'une heure environ et d'entretiens collectifs (focus groups) d'une durée d'une heure et demie. Les entretiens portaient sur les rôles joués par les écoles professionnelles et les entreprises. Ils s'intéressaient également aux composantes et contenus de l'identité professionnelle dans les deux professions retenues, ce aussi en fonction des filières de formation suivies (duale versus école à plein temps). Le traitement et l'analyse des données sont actuellement en cours. Les résultats seront disponibles dès mars 2008.

\section{Étude E2 : Jeunes en rupture dans la formation professionnelle initiale et construction identitaire.}

Equipe du projet : Jacqueline De Puy, Nadia Lamamra et Jonas Masdonati (IFFP).

La seconde étude porte sur les jeunes ayant arrêté prématurément leur formation professionnelle duale. Elle consiste en l'analyse qualitative de 45 entretiens semi-directifs (durée moyenne : une heure) menés avec des apprenants ayant quitté le système de formation durant la première année d'apprentissage. Les entretiens sont construits autour de trois grands thèmes : données socio-biographiques (parcours scolaire et de vie), raisons de la rupture (divisées en raisons de type personnel, relationnel ou systémique), 


\section{Encadré 3 (suite)}

situation actuelle (représentations des jeunes en rupture, gestion de la rupture et perspectives d'avenir). Les témoignages des jeunes font l'objet d'une analyse de contenu et sont également comparés aux explications évoquées par leurs anciens employeurs, interrogés par QCM (questionnaire à choix multiple). L'objectif de cette recherche est double. D'une part, elle va permettre de mieux comprendre le point de vue des jeunes ayant arrêté leur formation professionnelle duale : quelles sont les raisons évoquées? Quels processus ont abouti à la rupture? Comment les jeunes viventils et expliquentils ce qui s'est passé ? D'autre part, et sur la base des réponses à ces questions, elle aboutira à la proposition de mesures de prévention de la rupture d'apprentissage qui soient adaptées à la manière dont les jeunes la vivent ou l'expliquent. Le recueil, le traitement et l'analyse des données sont actuellement en cours. Les résultats seront disponibles dès janvier 2008.

Dans ce qui suit, nous proposons des réflexions sur les opportunités et les risques - en termes de construction identitaire - que présente le système d'apprentissage dual pour les apprenants. Ces réflexions théoriques sont appuyées et illustrées par des extraits d'entretiens issus du matériel recueilli dans le cadre de deux recherches en cours à l'IFFP, qui portent sur des apprenants dans leur première année de formation professionnelle duale. Dans la présente contribution ont été pris en considération dix entretiens individuels et trois entretiens collectifs de la première étude (apprenants poly-mécaniciens et leurs enseignants, filière duale), ainsi que quinze entretiens individuels de la deuxième étude (jeunes issus de différentes professions et ayant quitté prématurément leur formation).

La démarche est de type déductif. Les témoignages proposés ont été sélectionnés pour appuyer nos réflexions sur les atouts et les inconvénients de la formation professionnelle duale en matière de construction identitaire des apprenants. Néanmoins, seuls les témoignages qui constituaient une illustration parlante de l'ensemble du corpus ont été retenus dans cet article, fournissant ainsi une image fiable du matériel considéré. Notons que les exemples proposés proviennent principalement des discours des jeunes interrogés et mettent ainsi en évidence la perception qu'ils ont de leur situation et de celle du processus de formation. De manière accessoire, et afin d'éclairer ces témoignages lorsque cela s'avère nécessaire pour la compréhension d'une réflexion, des propos d'enseignants, de formateurs et d'experts sont également proposés. ${ }^{7}$

\section{UNE TRANSITION AMÉNAGÉE DE L'ÉCOLE AU TRAVAIL}

À propos des atouts identitaires du système dual, soulignons tout d'abord que celui-ci offre aux jeunes un espace institutionnalisé de transition entre deux mondes fort différents : celui de l'école obligatoire et celui du travail. En effet, le caractère hybride de la formation duale peut permettre aux apprenants d'entrer en contact de manière progressive avec le monde du travail et de quitter graduellement leur identité d'élève. Ainsi, comme le souligne CohenScali (2001b, p 199), l'alternance constitue une «transition aménagée » de l'école au travail. Dès lors, l'attrait du passage vers l'âge adulte, symbolisé par l'entrée sur le marché du travail, est évoqué fréquemment par les jeunes lorsqu'ils expliquent leur choix d'une formation professionnelle duale ${ }^{8}$ :

«J'avais envie de travailler! Vraiment envie de se sentir plus adulte, de se dire "voilà l'argent que tu gagnes, c'est toi qui dois travailler pour l'avoir!"

\footnotetext{
${ }^{7}$ Les citations se rapportant à la première étude seront suivies de la mention «E1 » et celles se référant à la seconde porteront la mention « $\mathrm{E} 2 »$.

${ }^{8}$ Pour faciliter la lecture, les extraits d'entretien ont été toilettés.
} 
[...] Je trouve que c'est une bonne chose, l'apprentissage, parce qu'en même temps on a de tout, on a les cours, la pratique, on a le travail [...]. [On entre] dans le monde d'adulte, oui! Parce que j'avais vraiment envie, besoin [de] ça! » (E2, ex-apprenant opticien, 17 ans).

En l'occurrence, entrer sur le marché du travail ne signifie donc pas uniquement gagner un salaire : cela peut surtout indiquer que les apprenants font partie d'un groupe, celui des personnes actives dans la société. Cette appartenance s'accompagne également des avantages qui y sont liés, en termes de capacité financière par exemple ou d'autonomie envers ses parents :

«Si [...] c'est un jeune qui veut se frotter à la réalité industrielle, qui a envie de quitter son uniforme de jeune de 16 ans et voir des personnes de tout âge travailler à ses côtés, qui désire avoir une autonomie maximale et pouvoir un petit peu se débrouiller, qui veut aussi toucher un peu d'argent, avoir une petite autonomie financière [...], un apprentissage dans le système dual est préférable. » (E1, enseignant d'une filière polymécanique).

Ensuite, et en particulier pour les apprenants ayant un passé scolaire difficile, une formation axée tant sur des cours théoriques que sur des enseignements pratiques sur le lieu de travail est l'occasion de construire une nouvelle forme d'estime de soi, basée davantage sur des compétences pratiques (Masdonati, 2007). De ce point de vue, l'entrée en formation professionnelle duale peut enclencher une dynamique identitaire de « restauration» (Barbier, 1996). Cette thèse est confirmée par Cohen-Scali (2001b), qui montre que, comparativement à une formation professionnelle entièrement scolaire, la formation en alternance permet aux jeunes de développer des représentations plus positives du monde du travail et de l'insertion, au travers d'une meilleure estime de soi et d'une augmentation du sentiment de compétence. La fierté de "savoir faire quelque chose», celle d'être apprécié, utile, reconnu comme capable de gagner sa vie peuvent découler, de manière générale, d'une formation professionnelle. Cette nouvelle estime de soi est aussi tributaire de la possibilité, offerte par l'apprentissage dual, de s'identifier à un corps de métier ou à une entreprise et de jouir d'une reconnaissance sociale en tant que membre de la « population active » (symbolisée notamment par la signature d'un véritable contrat de travail).

Le désir d'en finir avec l'école se situe donc au cœur des motivations de nombreux apprenants de première année interrogés, tant chez des jeunes ayant rompu leur formation professionnelle duale que chez ceux qui la poursuivent :

« Je suis pas un grand studieux à l'école [...] vouloir aller en cours [...] un jour c'est déjà pas mal, [...] je vais sur les bancs de l'école [...] et après c'est fini, je reprends plus ça, c'est sûr !» (E2, ex-apprenant opticien, 17 ans)

«Je voulais pas aller au gymnase ${ }^{9}$, parce qu'à l'école, déjà, je commençais à en avoir un peu marre. » (E1, apprenant polymécanicien, 17 ans)

«Il y a des apprentis, des jeunes, à qui la formation duale convient bien, parce qu'ils en ont, évidemment pour la plupart, "ras-le-bol" de l'école et ils ont qu'une envie: c'est de faire enfin quelque chose. » (E1, expert, administration cantonale)

Cependant, les jeunes débutant une formation professionnelle duale suivent tout de même des cours en école professionnelle, à raison d'un à deux jours par semaine. Cependant, la perception de cette formation est tout à fait différente de celle du cursus scolaire obligatoire. En effet, les cours professionnels ont bien plus de sens pour eux :

$A$ : «C'est pas du tout la même école »

$B: \ll L a ̀$, on travaille $[\ldots]$ pour nous »

$C$ : «Oui, pour ce qui nous intéresse. On travaille pour ce qui nous intéresse, tandis qu'à l'école, on faisait de ces trucs, euh...»

A : «Rien à voir »

$B$ : «Qui servaient à rien.» (E1, apprenants polymécaniciens)

Finalement, lors de la formation pratique en entreprise, les jeunes sont constamment en contact avec un formateur censé les initier au métier choisi. Or, cette personne ne joue pas un rôle strictement formateur,

${ }^{9}$ Dans le canton de Vaud, le gymnase correspond au lycée français, et débouche sur un baccalauréat. 
comme le dit Chaix (1993, p. 134). En effet, ces individus «mettent en æeuvre des dimensions de leur rôle qui ne sont pas seulement professionnelles ou pas liées uniquement aux situations de travail. Ce sont des rôles d'aînés par rapport à des jeunes, des rôles de co-formateurs, des rôles anticipant les qualifications des futurs professionnels et l'évolution de la profession, des rôles d'énonciateurs de la "pratique" ». En effet, le formateur peut également constituer une référence de poids et représenter un autrui significatif pour la construction de l'identité professionnelle de l'apprenant (Palmonari, 1993). La notion de transaction relationnelle (Dubar, 1996) ${ }^{10}$ semble adaptée pour qualifier l'importance du rôle de la personne formatrice au sein de l'entreprise, cette dernière jouant une fonction clé pour l'intégration et l'acceptation de l'apprenant au sein de l'organisation et contribuant par ce biais à sa socialisation professionnelle.

Les témoignages que nous avons recueillis semblent confirmer le fait que cette personne formatrice est souvent une figure de référence au niveau professionnel, mais également au-delà. Certains apprenants en attendent davantage qu'une relation employeuremployé, sans pour autant que la relation souhaitée soit clairement définie :

«Mon patron, [...] dès qu'on avait un moment, il m'emmenait dans des parcs, il m'expliquait tout plein de choses sur les arbres. D'ailleurs, tout ce qu'il m'a expliqué, je m'en rappelle encore, [...] c'était vraiment un super patron. Même mes problèmes professionnels, mes problèmes privés, je pouvais lui expliquer, il m'aidait et tout, et en plus, il avait à peu près les mêmes idées que moi, alors on s'entendait vraiment bien.» (E2, ex-apprenante paysagiste, 17 ans)

«Souvent, les maîtres d'apprentissage [les formateurs] n'ont pas qu'un rôle de maître d'apprentissage, il faut se substituer à des parents qui ne sont pas forcément toujours là, ou des situations familiales qui ne sont pas toujours idéales. » (E1, expert, administration cantonale)

\footnotetext{
${ }^{10}$ Selon Dubar, la transaction relationnelle consiste en l'articulation entre des identités attribuées ou proposées par autrui et des identités assumées ou incorporées par soi.
}

Un autre exemple est fourni par un entretien collectif mené avec des formateurs poly-mécaniciens. Dans ce cas aussi, le profil de la personne formatrice dépasse la simple fonction de transmission de compétences professionnelles. Si l'on en croit les propos recueillis, le formateur en entreprise doit être un exemple à tout point de vue :

« [Notre rôle est] de faire d'un enfant un adulte indépendant, qui soit capable de se subvenir à luimême. » (E1, formateur polymécanicien)

La première année est ainsi souvent une année d'adaptation qui permet de cerner les problèmes et de se mettre en phase avec l'apprenant. Il en est de même pour les enseignants d'écoles professionnelles qui, souvent issus du métier, tentent de transmettre, au travers de leur enseignement, non seulement un contenu lié à la branche, mais également un état d'esprit et une ambiance de travail, autant d'éléments qui participent d'une identité professionnelle.

\section{DE L'ÉLÈVE AU PROFESSIONNEL : UNE TRANSITION DIFFICILE}

Dans le contexte actuel, et en dépit des atouts évoqués, le système de formation professionnelle duale n'est pas sans défauts. Dans certaines circonstances, ceux-ci peuvent mettre en péril la construction identitaire des jeunes.

Premièrement, la transition entre l'école obligatoire et la formation professionnelle duale implique que le jeune en fin de scolarité trouve une place de formation en entreprise, idéalement dans la profession choisie. Or, ce processus est tributaire de la situation du marché des places d'apprentissage, ce dernier dépendant à son tour du marché du travail. Il n'est donc pas étonnant de constater régulièrement des décalages qualitatifs et quantitatifs entre les places d'apprentissage offertes par les entreprises et la demande des jeunes (LINK, 2005). De ce fait, des dynamiques de concurrence s'enclenchent parfois entre les jeunes en fin de scolarité obligatoire pour l'obtention d'une place de formation post-obligatoire. Ainsi, les jeunes plus démunis face à cette démarche ne parviennent pas à trouver une place d'apprentis- 
sage et demeurent une ou plusieurs années dans des solutions transitoires ( $10^{\mathrm{e}}$ année de formation, mesures dans le cadre de l'assurance-chômage, etc.) (Meyer, 2004). Or, le fait de ne pas trouver d'apprentissage et de devoir attendre ou faire face à des échecs répétés lors de candidatures pour un emploi peut être difficile à gérer et affaiblit l'estime de soi des jeunes, avant même que ceux-ci n'aient entamé leur formation professionnelle (Oser, Düggeli, Masdonati \& Gamboni, sous presse). En outre, l'offre de places ne correspond pas toujours aux désirs des jeunes (Meyer, Stalder \& Matter, 2003), qui sont souvent contraints d'opter pour des solutions de repli, ce qui peut également être vécu comme un échec :

«En fait, je suis sortie de l'école, j'avais déjà cherché dans la coiffure mais j'avais pas trouvé. Alors j'ai fait une année de stage dans un jardin d'enfants, parce que j'avais un peu des idées différentes de métiers, c'est un peu dur à choisir. Puis après j'ai commencé une école d'assistante en soins [...], mais le problème c'était qu'on faisait des stages en $E M S^{11}$, et je supportais pas [...], je tombais dans les pommes, j'étais tout le temps malade, enfin, c'était le métier qui n'allait pas, en fait. Donc, après j'ai cherché une place de coiffeuse et c'est là que j'ai commencé. » (E2, ex-apprenante coiffeuse, 18 ans)

«On en a tout plein qui sont là parce qu'ils n'ont rien trouvé d'autre! Alors, ils ont vu qu'il y avait un tas d'autres projets qui n'ont pas pu se concrétiser, et qui finalement sont venus dans un métier où il y a des disponibilités au niveau places d'apprentissage. Et ça, c'est pas à négliger. Il y en a quand même pas mal. Je pense, ça dépend des années, mais ça peut aller jusqu'à un bon tiers des élèves. » (E1, expert, doyen d'une filière poly-mécanique dans une école professionnelle)

De plus, la probabilité d'être parmi les jeunes qui ne trouvent pas de solution en sortant de l'école obligatoire est plus élevée pour les personnes d'origine étrangère, à bas statut socio-économique et à faible niveau scolaire (Haeberlin, Imdorf \& Kronig, 2004 ; Meyer, 2003). Ainsi ces jeunes peuvent développer déjà, lors de cette étape, un sentiment de stigmatisa-

11 Établissement médico-social pour les personnes âgées. tion et d'exclusion sociale lié à leur profil sociobiographique, ce qui n'est vraisemblablement pas sans conséquence sur leur définition d'eux-mêmes et sur leur relation avec le monde du travail.

Deuxièmement, même si l'apprentissage dual constitue un espace fondamental de transition entre l'école et le travail, il se déroule en contact direct avec le monde du travail et ses contraintes. Les apprenants doivent donc gérer l'adaptation à un rythme soutenu, exigeant sur le plan physique et intellectuel, puisqu'il s'agit de cumuler les cours et l'immersion dans un environnement de travail orienté vers la productivité. Nos interlocuteurs soulignent d'ailleurs cette productivité comme un des éléments essentiels à acquérir en cours de formation. Parfois, le poids des responsabilités qui en découlent peut être perçu comme trop lourd :

«C'est quand même une certaine responsabilité, une machine à disposition qui coûte deux millions de francs. [...] On a déjà eu des cas d'apprentis qui ont arrêté à cause de toute cette pression. Finalement, ils ont dit "je peux pas, j'ai peur! Je peux pas travailler sur ces machines". [...] L'apprenti, chez moi, le premier mois il fait déjà des pièces qu'il me faut pour la clientèle. » (E1, expert, chef d'entreprise)

Toujours est-il que ces contraintes, bien qu'elles soient fortes, sont rarement évoquées comme raison unique d'une rupture de contrat d'apprentissage. Il apparait cependant que le contact avec le monde du travail, même s'il est souhaité, peut constituer un élément déstabilisant pour un jeune déjà fragilisé. On peut donc faire l'hypothèse que l'espace de transition offert par l'apprentissage dual ne permet pas toujours aux jeunes de tester des nouvelles identités, de faire des erreurs, de se réorienter, bref, de se construire progressivement en tant que travailleur sans que cela n'implique une remise en question globale et déstabilisante du point de vue identitaire (Bourgeois, 1996; Dubar, 2000). Ainsi, le passage entre une identité d'élève et une identité de professionnel n'est pas toujours aisé :

"Quand on est à l'école on nous dit, "ouais, tu vas voir, tu vas entrer en apprentissage, ça va être dur et tout". Puis quand [on] est en plein dedans, on finit la semaine, on se dit: "j'étais bien à l'école, quand même"...» (E2, ex-apprenante paysagiste, 17 ans) 
Cette ambiguité, qui tient peut-être au système dual lui-même, fait que les témoignages sont parfois contradictoires. Les jeunes veulent être considérés comme des professionnels, ce qui est une image valorisée, mais en même temps ils souhaitent être des jeunes en formation, soutenus et encadrés par les adultes.

En troisième lieu, il nous semble que le rôle identitaire de la figure du formateur en entreprise est sousestimé. En effet, les extraits laissent apparaître des attentes envers les formateurs très fortes chez certains apprenants. Les formateurs sont souvent perçus, ainsi que nous l'avons vu, comme des adultes de référence, mais aussi des personnes desquelles on attend une proximité amicale et de conseil :

«Il s'est jamais occupé de moi! Rien! Je pouvais pas lui poser de questions. » (E2, ex-apprenant opticien, 17 ans)

«La maître d'apprentissage, je la vois, c'est "bonjour", mais rien d'autre. » (E2, ex-apprenante employée de commerce, 17 ans)

«Avec lui, je sais pas, c'était plutôt, [...] oui, on parlait pas trop, des fois il me posait des questions sur ma vie et tout, il me répondait, ça me faisait plaisir. Lui aussi me parlait des sports qu'il faisait, deux ou trois petits trucs comme ça, mais c'était peutêtre 10 minutes en huit mois!» (E2, ex-apprenant informaticien, 18 ans)

Sur la base de ces témoignages, nous supposons que, à cause d'une formation très condensée et de l'absence d'un encadrement régulier (notamment sous forme de coaching ou de supervision), les responsables de formation en entreprise ne mesurent pas toujours la portée de leur rôle. Les résultats de l'étude de Cohen-Scali (2001b) portant sur les attitudes des apprenants semblent aller dans le même sens. En effet, les effets positifs sur les apprenants en formation par alternance sont largement tributaires de la qualité de l'accueil et de l'encadrement au sein de l'entreprise.

Un dernier point faible est étroitement lié au précédent et porte sur le fait que, globalement, le système dual semble incapable de gérer le côté le plus délicat du développement identitaire, à savoir la crise. Ainsi, nous constatons l'absence de mesures institutionnelles d'envergure nationale permettant de prévenir et de gérer la rupture d'apprentissage ( $c f$. note 6). Or, ce phénomène peut être considéré comme la manifestation explicite de la mauvaise résolution d'une crise identitaire (Lamamra \& Masdonati, 2006). Dans leur étude qualitative auprès de 72 jeunes sans qualification, Eckmann-Saillant, Bolzman et de Rham (1994) relèvent par exemple que les jeunes ayant quitté prématurément le système de formation professionnelle sont conscients de subir une stigmatisation du fait de leur situation précaire et de la difficulté qu'ils auront pour se réinsérer et déclarent ne pas faire pleinement partie de la société. De plus, dans une enquête auprès de 200 jeunes en rupture d'apprentissage, Ferron, Cordonier, Schalbetter, Delbos-Piot et Michaud (1997) montrent que l'arrêt de formation augmente les probabilités de consommation de produits psychotropes, ce qui peut être vu comme une manifestation d'un malaise psychique ou social. Par ailleurs, dans nos recherches, de nombreux éléments renvoient à une souffrance vive des apprenants en rupture de formation. Nous avons dès lors considéré la rupture accompagnée de souffrance comme l'aboutissement d'une crise (Lamamra \& Masdonati, 2006) :

«Je dormais plus et j'avais une fatigue mentale, donc je dormais, mais je me reposais pas, et j'avais beaucoup de peine à dormir, je faisais des cauchemars toutes les nuits, [...] je mangeais plus, enfin, j'avais de la peine à manger: démoralisée, quoi! J'avais plus goût à rien, je voyais vraiment la vie en noir! J'avais plus aucune motivation, j'avais plus envie de rien faire. » (E2, ex-apprenante gardienne d'animaux, 17 ans)

«Je disais à ma mère, "j'en peux plus! Tous les jours j'en peux plus!” Je m'énervais avec elle! "J'en peux plus, je vais me casser!" Oui, je me demande comment j'ai fait pour tenir comme ça!» (E2, ex-apprenant employé de commerce, 20 ans)

« Je rentrais à la maison, j'avais mal à l'estomac, je me rendais malade, j'avais plus envie d'aller travailler [...], j'étais juste mal! [...] J'avais pas envie de me lever pour aller travailler. » (E2, exapprenant opticien, 17 ans)

«Puis du coup je suis partie en dépression. Donc [...] je mangeais plus, je me mutilais.» (E2, exapprenante employée de commerce, 17 ans) 
Précisons toutefois que la souffrance et la crise peuvent être provoquées par un questionnement identitaire au sens large (orientation et choix professionnel, adaptation au monde des adultes et au monde professionnel, problèmes dans d'autres sphères de vie), mais également par d'autres situations ou événements externes à l'individu. Par exemple, nos témoignages révèlent aussi que la crise est parfois liée à un milieu de travail pénible (problèmes relationnels, pressions, harcèlement).

En somme, les apprenants sont amenés à consentir des efforts importants pour s'adapter à un système et un contexte qui exigent beaucoup de leur part. Lorsque ce parcours est réussi, il contribue vraisemblablement à forger une identité professionnelle valorisante et valorisée. Mais les principales limites du système tiennent peut-être dans la gestion des «pannes » et des situations de crise, lesquelles résultent d'une conjonction de facteurs tant personnels que contextuels.

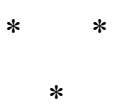

Revisiter le système de formation professionnelle duale dans une perspective identitaire amène à brosser un tableau en demi-teinte. En effet, les réflexions et les extraits proposés laissent d'une part supposer que ce type de formation peut contribuer de manière décisive à la construction identitaire des apprenants. L'apprentissage dual est susceptible de permettre en effet aux jeunes d'affronter la transition entre le monde scolaire et le monde du travail de manière graduelle, favorisant ainsi la transition entre une identité d'élève et une identité de professionnel. Par ailleurs, parce qu'il valorise non seulement les savoirs mais aussi les savoir-faire, il offre aux jeunes en difficulté scolaire la possibilité de dépasser une expérience négative de l'école. Enfin, la présence d'un formateur en entreprise peut favoriser la projection des jeunes dans un modèle professionnel, élément indispensable dans la construction identitaire.

Cependant, nos témoignages révèlent que l'apprentissage dual présente plusieurs limites non négligeables. Au vu des impacts que le déroulement de la formation peut avoir sur les trajectoires profession- nelles et de vie des personnes, ces limites méritent une attention toute particulière. Tout d'abord, les tensions liées au passage de l'école obligatoire à la formation professionnelle (qui se déroule dans un vrai marché régi par les lois de l'offre et de la demande) laissent craindre qu'un nombre important de jeunes soit confronté à une véritable remise en question avant même d'entrer en apprentissage dual. Ensuite, l'aspect progressif de l'entrée en contact avec le monde du travail n'est souvent qu'apparent, les apprenants étant dans la majorité des cas soumis pleinement aux logiques du monde du travail dès leur formation professionnelle. Par ailleurs, dans plusieurs situations rencontrées dans nos recherches, le formateur en entreprise n'est de facto pas en mesure de jouer le rôle identitaire positif évoqué cidessus, ce qui peut engendrer des situations délicates du point de vue de la construction identitaire des apprenants. En dernier lieu, le système de formation professionnelle duale n'est pas en mesure de gérer certaines situations de crise identitaire. De ce point de vue, le manque de moyens de prévention ou de gestion des ruptures de contrat d'apprentissage est symptomatique d'une lacune qu'il nous semble urgent de combler. Ceci d'autant plus que, sans formation post-obligatoire, les risques de précarité et de pauvreté qui y sont associés sont importants (Meyer, 2003 ; Volken \& Knöpfel, 2004).

Ces tendances et ces réflexions mériteraient d'être explorées plus avant dans les analyses ultérieures de nos données et vérifiées dans d'autres études. Il serait en effet souhaitable non seulement d'approfondir le rôle identitaire de la formation professionnelle duale, mais aussi de compléter nos analyses avec des données représentatives. Des recherches davantage quantitatives permettraient notamment de répondre aux questions suivantes: quelle est la proportion des apprenants dont la construction identitaire est étroitement associée à la manière dont se déroule leur formation professionnelle ? Y a-t-il un profil sociobiographique d'apprenants plus sensibles aux incidences de la formation professionnelle sur leur construction identitaire? Constate-t-on des différences selon le métier choisi ? Répondre à ces questions permettrait ainsi de mieux cibler les mesures de soutien et de prévention au sein de la formation professionnelle duale. 
Par ailleurs, une démarche de type longitudinal permettrait de mettre en évidence les stratégies et les démarches mises en place par les jeunes pour «se construire » en composant avec les atouts et les inconvénients de ce système de formation. Il s'agirait, en d'autres termes, de considérer les apprenants davantage comme des acteurs de leur construction identitaire et d'appréhender cette dernière dans une perspective temporelle.

Si les constats issus de cet article devaient être confirmés dans la suite de nos travaux ou par d'autres études, deux types de perspectives concrètes seraient envisageables. D'une part, il y aurait vraisemblablement lieu de mieux valoriser et développer les atouts du système dual. Par exemple, le rôle de la formation professionnelle duale en tant que moyen de revaloriser la relation à l'institution scolaire pourrait être accentué par la mise en valeur du sens et de la pertinence d'une formation professionnelle dans le contexte socio-économique actuel. Il serait notamment concevable de proposer une sensibilisation aux enseignants et aux élèves en fin de scolarité obligatoire qui porterait sur l'utilité et les avantages de ce type de formation. En début d'apprentissage dual, il conviendrait également d'expliciter aux apprenants les phases d'adaptation qui les attendent, tout en montrant les passages obligés d'une socialisation professionnelle. Enfin, il serait souhaitable aussi d'informer les jeunes de leurs droits, afin qu'ils sachent ce qui peut leur être demandé ou non, cela notamment en termes de prévention des risques professionnels.

D'autre part, il conviendrait de prévoir des mesures permettant de limiter les inconvénients identitaires précédemment cités. Premièrement, des mesures sont à envisager afin d'établir un meilleur équilibre entre offre et demande de places d'apprentissage. Ceci éviterait aux jeunes en transition de se retrouver au chômage dès l'âge de 15-18 ans. Le renforcement de la formation professionnelle en école à plein temps - pendant les périodes de pénurie de places d'apprentissage ou pour les métiers pour lesquels la demande de places dépasse régulièrement l'offre - et une sollicitation plus poussée des entreprises à former des apprenants (soutien financier, accompagnement, valorisation), sont deux exemples de mesures structurelles et institutionnelles envisageables. Deuxièmement, les élèves en école obligatoire pourraient être davantage sensibilisés aux changements qu'ils vont rencontrer lors de l'entrée en formation professionnelle. À cela pourrait s'ajouter la possibilité, pour les apprenants en début de formation, d'évoluer dans un contexte de formation moins soumis aux attentes de performance et de productivité des entreprises. La première année de formation professionnelle pourrait par exemple avoir lieu davantage en école qu'en entreprise. Soulignons que cette formule est testée actuellement dans certaines professions qui ont introduit un modèle dégressif, le temps en école diminuant entre la première et la troisième année de formation. Troisièmement, les formateurs pourraient être davantage sensibilisés à leur rôle identitaire et aux attentes des apprenants (Chaix, 1993). On pourrait mettre à leur disposition des ressources afin de favoriser le dialogue apprenants-formateurs et de les aider à gérer des situations délicates. Dans le même ordre d'idées, ces ressources peuvent aussi jouer un rôle de prévention et d'accompagnement des apprenants, des enseignants et des formateurs lors de l'apparition de crises identitaires chez les jeunes en formation. Des mesures développées actuellement par la Confédération et permises par la nouvelle loi sur la formation professionnelle vont justement dans ce sens. Citons par exemple la possibilité offerte aux jeunes en transition entre école obligatoire et formation professionnelle duale de bénéficier d'un accompagnement individuel sous forme de coaching (suivi personnalisé dans la durée) ou de case management (coordination des mesures et institutions déjà impliquées dans le suivi du jeune).

En somme, un large éventail de mesures découle d'une confirmation des réflexions et témoignages proposés ci-dessus. Certaines de ces mesures sont actuellement discutées et testées par les instances cantonales et fédérales. D'autres mériteraient davantage de réflexion et de volonté institutionnelle et politique. 
Bibliographie

Barbier J.-M. (1996), «De l'usage de la notion d'identité en recherche, notamment dans le domaine de la formation », Éducation Permanente, 128(3), pp. 11-26.

Berthoud A. \& Michaud P.A. (1997), Accompagnement et prévention des ruptures d'apprentissage, Lausanne : Institut universitaire de médecine sociale et préventive.

Bourgeois E. (1996), «Identité et apprentissage », Éducation Permanente, 128(3), pp. 27-35.

Castel R. (1995), Métamorphose de la question sociale. Une chronique du salariat, Paris, Fayard.

Chaix M.-L. (1993), Se former en alternance : Le cas de l'enseignement technique agricole, Paris, L'Harmattan.

Cohen-Scali V. (2000), Alternance et identité professionnelle, Paris, Presses Universitaires de France.

Cohen-Scali V. (2001a), « Le rôle des composantes de la personnalité dans le processus de transition de l'école au travail », Connexions, 76(2), pp. 41-59.

Cohen-Scali V. (2001b), « Les attitudes à l'égard de l'insertion professionnelle d'apprentis de l'enseignement supérieur », L'Orientation Scolaire et Professionnelle, 30(2), pp. 173-202.

De Puy J., Castelli-Dransart D.-A., Gay-des-Combes B., Perriard V. \& Zbinden Sapin V. (2006), "Young Trainees and Professional Identity in Switzerland: Pride or Prejudice", Sociological Abstracts, 2006S00507.

Dubar C. (1996), La socialisation : construction des identités sociales et professionnelles, (2e éd.), Paris, Armand Colin.

Dubar C. (2000), La crise des identités (2e éd.), Paris, Presses Universitaires de France.
Dubs R. (2006), Rapport d'expertise sur les questions concernant la formation professionnelle en Suisse, Bern, HEP.

Eckmann-Saillant M., Bolzman C. \& de Rham G. (1994), Jeunes sans qualification: trajectoires, situations et stratégies, Genève, Les Éditions IES.

Erikson E. H. (1972), Adolescence et crise (Nass J. \& Louis-Combet C., Trad.), Paris, Flammarion.

Ferron C., Cordonnier D., Schalbetter P., Delbos-Piot I. \& Michaud P. A. (1997), La santé des jeunes en rupture d'apprentissage, Lausanne : Institut universitaire de médecine sociale et préventive.

Flammer A. \& Alsaker F. (2002), Entwicklungspsychologie der Adoleszenz, Bern; GöttingenToronto, Seattle: Hans Huber.

Haeberlin U., Imdorf C. \& Kronig W. (2004), Chancenungleichheit bei der Lehrstellensuche: Der Einfluss von Schule, Herkunft und Geschlecht, Leitungsgruppe des NFP 43, retreived June 01, 2005, from http://www.nfp43.unibe.ch.

Hanhart S. (2006), « Marché de l'apprentissage et pouvoirs publics », Bulletin de la CIIP, 19, pp. 8-9.

Kirpal S. (2006), « Les identités au travail dans des perspectives comparatives: Le rôle des variables contextuelles nationales et sectorielles », Revue Européenne de Formation Professionnelle, 39(3), pp. 24-50.

Lamamra N. \& Masdonati J. (2006), Jeunes en difficulté dans la formation professionnelle: Une question identitaire? Paper presented at the $14^{\mathrm{e}}$ congrès de l'AIPTLF, Hamammet, Tunisie.

LINK (2005), Baromètre de l'apprentissage: Rapport condensé, extrait de www.bbt.admin.ch, 19 juillet. 
Masdonati J. (2007), La transition entre école et monde du travail: Préparer les jeunes à l'entrée en formation professionnelle, Bern, Peter Lang.

Meyer T. (2003), When being smart is not enough: institutional and social access barriers to upper secondary education and their consequences on successful labour market entry. The case of Switzerland, Paper presented at the 2003 Workshop of the European Research Network on Transitions in Youth, Funchal, Madeira.

Meyer T. (2004), Wie weiter nach der Schule? L'école... et après? Leitungsgruppe des NFP 43, retreived February 08, 2005, from http:// www.nfp43.unibe.ch

Meyer T., Stalder B. E. \& Matter M. (2003), Bildungswunsch und Wirklichkeit. Neuchâtel: Bundesamt für Statistik, Schweizerische Konferenz der kantonalen Erziehungsdirektoren.

Moser U. (2004), Jugentliche zwischen Schule und Berufsbildung: Eine Evaluation bei Schweizer Grossunternehmen unter Berücksichtigung des internationalen Schulleistungsvergleichs PISA, Leitungsgruppe des NFP 43, retreived June 01, from http:// www.nfp43.unibe.ch.

OFFT (2006), La formation professionnelle en Suisse en 2006, extrait de www.bby;admin.ch/berufsbi/ publikat, 19 juillet 2006.

Oser F., Düggeli A., Masdonati J. \& Gamboni E. (sous presse), Die Zeitbombe des "dummen» Schülers: Eine Interventionsstudie zur Erhöhung von Resilienz bei drohender oder erfahrener Erwerbslosigkeit unterqualifizierter Jugendlicher.

Palmonari A. (Ed.) (1993), Psicologia dell'adolescenza, Bologna: Il Mulino.

Poglia E. (1999), «Globalisation : terrain miné ou terrain fertile pour l'éducation? », in Carton M., Hanhart S., Perez S., Poglia E. \& Terrier J. (Éds.), Globalisation économique et systèmes de formation en Suisse, Genève, Cahiers de la section des sciences de l'éducation, pp. 25-45.
Rastoldo F. (2006), « Les élèves de la 9e s'expriment sur leur choix d'orientation », Panorama, 1, pp. 19-20.

Rifkin J. (1996), La fin du travail, Paris, La Découverte.

Rochat S. \& Lamamra N. (2004), «La socialisation des apprenti-e-s », Panorama, 4, pp. 21-22.

Ruano-Borbalan J.-C. (2004), « La construction de l'identité ", in Halpern C. \& Ruano-Borbalan J.-C. (Éds.), Identité(s), Auxerre, Sciences Humaines Éditions, pp. 1-10,

Schulenberg J., Maggs J. L. \& Hurrelmann K. (Eds.). (1997), Health Risks and Developmental Transitions During Adolescence, Cambridge, Cambridge University Press.

Sennett R. (2000), Le travail sans qualités: Les conséquences humaines de la flexibilité, Paris, Éditions Albin Michel.

Sigerist P. \& Schwaab J.-C. (2006), « 10000 nouvelles places d'apprentissage sont nécessaires maintenant, pour que les jeunes ne se retrouvent pas sur la touche », extrait de www.uss.ch, 15 février 2007.

Stalder B. E. \& Schmid E. (2006a), Lehrvertragsauflösungen, ihre Ursachen und Konsequenzen: Ergebnisse aus dem Projekt LEVA, Bern, Bildungplanung und Evaluation der Erziehungsdirektion des Kantons Bern.

Stalder B. E. \& Schmid E. (2006b), «Raisons et conséquences des abandons d'apprentissage », Panorama, 2, pp. 3-14.

Troger V. (2003), « La formation professionnelle des jeunes en question », Sciences Humaines, 140, pp. 16-20.

Volken J. S. \& Knöpfel C. (2004), Risque de pauvreté N. 1: une mauvaise formation! Les parcours des personnes touchées par la pauvreté en Suisse, Lucerne, Éditions Caritas.

Zittoun T. (2006), Insertions. A quinze ans, entre échec et apprentissage, Bern, Peter Lang. 


\title{
Résumé
}

\section{Les enjeux identitaires de la formation professionnelle duale en Suisse : un tableau en demi-teinte}

\author{
Jonas Masdonati, Nadia Lamamra, Benoît Gay-des-Combes, Jacqueline De Puy
}

Cet article questionne le rôle de la formation professionnelle duale dans la construction identitaire des apprenants. Le matériel utilisé pour répondre à ce questionnement provient de deux recherches en cours à l'Institut fédéral des hautes études en formation professionnelle, qui portent sur les apprenants en première année de formation. Les premiers résultats indiquent que ce système de formation présente aussi bien des forces que des faiblesses quant à sa contribution au développement identitaire des apprenants. Cet état des lieux débouche sur des perspectives concrètes visant à renforcer les atouts et à remédier aux inconvénients de la formation professionnelle duale, telles que l'aménagement de la transition entre école et formation professionnelle ou la valorisation du rôle des formateurs en entreprise.

\section{Mots clés}

Formation professionnelle, Formation en alternance, Identité professionnelle, Suisse

Journal of Economic Literature: M 53 Training ; I 21 Analysis of Education 


\section{Informations}

\section{sociales}

Créée en 1946, la revue Informations sociales est éditée par la Caisse nationale des allocations familiales (CNAF). Outil de réflexion et d'information pour les praticiens du social, Informations sociales est également un lieu de synthèse et de débat pour les chercheurs et les décideurs.

La revue est construite sur des dossiers thématiques qui associent les spécialistes et les acteurs d'une question sociale. Cette revue, avec six numéros dans l'année à compter de 2008, accompagne acteurs, chercheurs et décideurs dans leurs analyses, leurs expertises et leurs actions.

Pour découvrir la revue, nous vous proposons de choisir un exemplaire à titre gracieux parmi nos dernières livraisons :

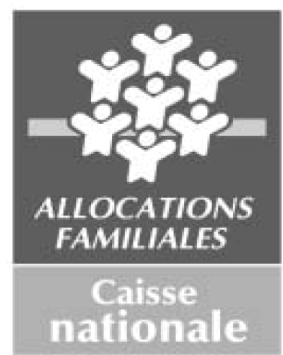

$$
\begin{aligned}
& \mathrm{n}^{\circ} 140 \text { - L'enfant dans le système administratif et judiciaire } \\
& \mathrm{n}^{\circ} 141 \text { - Familles et quartiers sensibles } \\
& \mathrm{n}^{\circ} 142 \text { - Protection sociale et emploi } \\
& \mathrm{n}^{\circ} 143 \text { - Politiques familiales et sociales au Québec } \\
& \mathrm{n}^{\circ} 144 \text { - L'amour et la famille }
\end{aligned}
$$

Pour recevoir votre exemplaire, vous pouvez soit faire votre demande directement par mail à l'attention de ysabelle.michelet@cnaf.fr, soit par un courrier adressé à la CNAF.

Retrouvez la présentation des numéros sur www.cnaf.fr, rubrique Publications

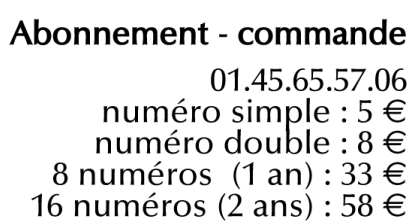

16 numéros $(2$ ans $): 58 €$

\section{CNAF}

Informations sociales - Y. Michelet

32 avenue de la Sibelle

75685 Paris cedex 14

Tél. 01.45.65.52.62 